\title{
Knowledge, Beliefs and Practices of Caregivers Regarding Home Based Bedsore Care in Chandigarh, North India
}

\author{
Upasana Sharma, Sukhpal Kaur, Amarjeet Singh
}

\section{ABSTRACT}

Background: Bedsores are quite frequent in bedridden cases. Caregivers have an important role in the management of such cases.

Objectives: To assess the knowledge, beliefs and practices of caregivers regarding bedsore care practices and management.

Materials and methods: A cross-sectional study was conducted in Chandigarh from September to November 2011. Caregivers were interviewed about the knowledge, attitude and practice regarding care of bedridden patients and their problems like bedsores.

Results: Data was collected from 42 bedridden cases ( 22 males and 20 females). Age was 18 to 90 years. Bedsores were found in 10 bedridden cases. Most common underlying diagnosis was neurological disease in 24 cases. Twenty-nine patients consulted government hospitals. Earlier episode of bedsore was experienced in about 14 cases. In 24 cases bed sheet was changed daily. In 11 cases, for more than two times patient had to wait for more than half an hour if urine/stools were passed in bed. In six cases unqualified practitioners were also consulted. Thirty-seven of them complied with the physician's advice. Seventeen of the caregivers were aware of the warning signs of development of bedsores. Exercises were not performed in 26 cases. Different dressing regimes were reported viz til oil, desi ghee, platelet ointment, paraffin wax, etc.

Conclusion: Quality of care of bedridden was not good. There is a need for formal training for the caregivers as most of them were untrained. Evaluation of various dressing regimes for bedsores is needed.

Keywords: Bedsores, Family caregivers, Knowledge, Bedsore care practices.

How to cite this article: Sharma U, Kaur S, Singh A. Knowledge, Beliefs and Practices of Caregivers Regarding Home Based Bedsore Care in Chandigarh, North India. J Postgrad Med Edu Res 2013;47(3):138-143.

Source of support: Nil

Conflict of interest: None declared

\section{INTRODUCTION}

The process of human aging has been a topic of discussion in nearly every country in the world, since the life expectancy of people has increased significantly. This is due to improved conditions of life, sanitation, work and education, as well as technological conditions that made it possible for people to live longer and with more quality.

The recent demographic trend shows that in 1950, 5.6\% of the India's population was classified as elderly, which by 1990 rose to $7.1 \%$, and by 2025 it is likely to reach $12.3 \%$. In the industrialized countries of the West, the elderly population in the 1990 s was 7 to $15 \%$ of the total population, and is expected to reach $25 \%$ by the year $2020 .^{1}$

Many of the elderly have various degrees of disability. They are often dependent on others for their activities of daily living. Some of them remain bedridden due to various causes. As the age advances there is decline in physical function which leads to a loss of independence as well as the person become susceptible to both the acute and chronic health problems including arthritis, heart diseases, hypertension, bowel and urinary incontinence, dementia and skin diseases, etc. Thus, the increase life expectancy rather has an element of morbidity and disability linked with it. ${ }^{2}$

Many elderly remain bedridden in last years of their lives. Apart from this many young people also become bedridden due to various diseases like paralysis. Bedsores are the most prevalent wound and common complications among the bedridden patients. Because of the impaired mobility and altered nutritional pattern which may be related to the disease condition or to the aging process as such, the elderly people are at high risk of developing bedsores. It has been estimated that more than $50 \%$ of the bedridden patients develop bedsores. ${ }^{3}$ The incidence of bedsores has been reported to be 0 to $17 \%$ in home care settings. ${ }^{4}$

Most of these patients are cared for at home, with family members and friends providing the bulk of the care. This situation has lead to reorientation of health care systems in the developed countries from hospitals and nursing institutions to the domiciliary care arrangements that the chronically disabled prefer and use. Shifting of long-term care provision in rich societies from institutionalized care to the home has brought into focus the quality of care available to the patients at home. While data about the quality of care being provided in hospitals, nursing homes, residential institutions is available directly or indirectly, data about the quality of long-term care (LTC) provided to the severely disabled at their own homes is difficult to gather because of the multiple sociodemographic and interpersonal factors involved.

Against this background, in the present study, we have tried to assess the knowledge, beliefs and practices of caregivers regarding home-based bedsore care practices and management in Chandigarh.

\section{MATERIALS AND METHODS}

This cross-sectional study was conducted between September 2011 and November 2011 in the Chandigarh city. 
A community sample of 42 bedridden cases above the age of 12 years was examined and their caretakers were interviewed regarding their knowledge, practices and beliefs about bedsore care. This was done to gain a better insight of the parameters of the knowledge and beliefs of the caregivers regarding care of bedridden at home. No sampling was done to select the cases.

To enlist such cases, data on bedridden cases from various wards of PGIMER was accessed. Those residing in Chandigarh were contacted to know their current status. Information about such families was also sought from various other sources also, namely, personal acquaintances of the investigator, senior citizen associations of Chandigarh, local private practitioners, physiotherapists, masseurs, chemists, and suppliers of physical appliances, and so on. The addresses of the families were noted. The investigator visited these families. The subjects and their caregivers were interviewed individually. EPI info version 2000 was used for the analysis. Proportions were used for the interpretation of data. The subjects were informed about the purpose of study and consent was taken. Proxy consent was taken from the key care provider or the head of family, in cases where they were unable to communicate.

\section{Operational Definitions}

Bedridden case: This included all cases above 12 years of age who had been confined to bed for 15 days or more, for $90 \%$ of the time during the day and who were unable to get out of bed without assistance.

Key caregiver: The person in the family who was primarily responsible for the care of the index case.

\section{Exclusion Criteria}

All ambulatory cases were excluded, i.e. those who could get off the bed without assistance or those who could walk without assistance.

\section{RESULTS}

About 22 (52\%) were males and 20 (48\%) were female bedridden cases. Twenty-nine of them were married, six of them were unmarried and divorced each and one was separated. Seventeen of them had education up to graduation level and above. Maximum (78.5\%) were either not working or retired. About $4.7 \%$ each belonged to service and agricultural background. There were three students among the bedridden cases.

Maximum (95\%) of the patients were conscious. Neurological (57.1\%) and musculoskeletal (19.1) causes were the most common reasons of bedridden. PGIMER (52.4\%), followed by private doctors (21.4\%) were the most common source of medical consultation. One patient was on gastronomy feed and diabetic diet each. Two were on NPO-IV fluid.

In many (69\%) cases, patients needed some sort of assistance for changing the position. In 15 cases, frequency of position change was 2 hourly as recommended. In four cases position of the patient was seldom changed. The most common type of mattress that was found to be used was Rexine type (69\%), followed by air mattress (19\%). Bed sheet was changed daily in $57.1 \%$ cases whereas almost an equal number, i.e. around 9 reported to have been changing the bed sheet on alternate days or once a week. It was changed as soon as the bed got wet due to passage of urine or stool, in one case only. Bad smell around the patient was always present in five cases whereas in three cases, it was present sometimes. In 11 cases patients had to wait for more than half an hour for less than two times in a week to be cleaned for excretions on the bed whereas five cases reported that they had to wait most of the times for the same. Interestingly, majority (59.5\%) of them failed to comment when asked the same question (Table 1).

Bedsores were present in 10 bedridden cases during the period of study. Around 14 other had experienced an earlier episode of bedsores. Hence, the lifetime prevalence rate was reported to be 24, i.e. they had experienced an episode of bedsores in their entire lifetime ever.

Eighty-six percent of the cases had consulted someone other than the formally trained medical practitioners. Thirtyseven patients said that the physicians' advice was being followed for them.

Family member was the key caregiver in 36 cases whereas hired help was involved in six cases.

The average monthly expenditure spent for the care of bedridden patients was in the range of 1,001 to 5,000 in most of the cases (71.4\%). The major financial assistance, if taken, from nonfamily members was mostly from relatives (4.8\%). But it was seen that usually (88.1\%) people did not seek any financial help from anyone. In one case, free treatment was being received from a government hospital. Various kinds of modifications were done in the home/ environment (69\%) to enable the bedridden patient to function properly. Mostly modifications were done in the form of readjustment of furniture, chair with toilet or bedpan, mechanized bed and bed tables.

On observation it was found that correct position was ensured in 37 cases, i.e. large pillows were placed under the head and back and head end of the bed was little elevated in few cases. Exercises were performed in 16 cases only. Out of those 16, principles of exercising were being followed in 11 cases only. 
Twenty-three caregivers were completely aware of the various warning signs of the bedsores viz pain at the site, areas of duskiness or darkness, discharge, etc. Six of them were partially correct in recognizing the warning signs. Again, 23 of them knew that to prevent bedsore, bedridden patient should be dragged slowly or lifted for changing the position, whereas 17 of them were somewhat aware of the technique of changing the position. When asked that could friction be also a cause of bedsore, 22 were partially correct in telling so. Only seven knew that friction could also be a cause of bedsore formation. Very few (4) of them knew that right kind of diet could facilitate bedsore healing. Twenty-three believed that bedsores could not be prevented at all. Twelve caregivers agreed to the fact that overweight patients have greater chances of developing bedsore whereas 25 of them believed the opposite of that. Twenty of them believed that while dressing the wound of the patient, hand washing is not necessary if the gloves are worn. Only 10 caregivers considered it important to do so. There was

\begin{tabular}{|c|c|}
\hline Variables & $N(42)$ \\
\hline \multicolumn{2}{|l|}{ Position change } \\
\hline $\begin{array}{l}\text { Self } \\
\text { Assisted }\end{array}$ & $\begin{array}{l}13(31 \%) \\
29(69 \%)\end{array}$ \\
\hline \multicolumn{2}{|l|}{ Frequency of position change } \\
\hline $\begin{array}{l}\text { Less than hourly } \\
\text { Every } 2 \text { hourly } \\
2-4 \text { hourly } \\
4-6 \text { hourly } \\
\text { Seldom change }\end{array}$ & $\begin{array}{r}10(23.8 \%) \\
15(35.7 \%) \\
10(23.8 \%) \\
3(7.1 \%) \\
4(9.5 \%)\end{array}$ \\
\hline \multicolumn{2}{|l|}{ Type of mattress } \\
\hline $\begin{array}{l}\text { Rexine } \\
\text { Air mattress } \\
\text { Any other }\end{array}$ & $\begin{array}{r}29(69 \%) \\
8(19 \%) \\
5(12 \%)\end{array}$ \\
\hline \multicolumn{2}{|l|}{ Frequency of bed sheet change } \\
\hline $\begin{array}{l}\text { Daily } \\
\text { Alternate days } \\
\text { Once/week } \\
\text { As frequently as I wet my bed }\end{array}$ & $\begin{array}{r}24(57.1 \%) \\
9(21.4 \%) \\
8(19.5 \%) \\
1(2.3 \%)\end{array}$ \\
\hline $\begin{array}{l}\text { Bad smell around the patient } \\
\text { Yes } \\
\text { Sometimes }\end{array}$ & $\begin{array}{r}5(12 \%) \\
3(7 \%)\end{array}$ \\
\hline \multicolumn{2}{|c|}{$\begin{array}{l}\text { Frequency for which the patient had to wait for more } \\
\text { than half an hour in a week to be cleaned for } \\
\text { excretions on the bed }\end{array}$} \\
\hline $\begin{array}{l}\text { No comments } \\
\text { Most of the times } \\
\text { Less than five times } \\
\text { Less than two times }\end{array}$ & $\begin{array}{r}25(59.5 \%) \\
5(11.9 \%) \\
1(2.3 \%) \\
11(26.1 \%)\end{array}$ \\
\hline \multicolumn{2}{|c|}{$\begin{array}{l}\text { Observation of caregivers practices regarding } \\
\text { bedsore care }\end{array}$} \\
\hline Correct position ensured & $37(88 \%)$ \\
\hline Performs exercise & $16(38 \%)$ \\
\hline Follows principles of exercising & $11(26 \%)$ \\
\hline Gentle massage & $19(45 \%)$ \\
\hline
\end{tabular}

misconception among many regarding massage in bedridden patients. Twenty-six believed that the reddened bony areas should be massaged. Again, maximum (32) of them believed that salt water solution is not to be used for cleaning the wounds. Twenty-six caregivers knew that cotton clothes should be used for bedridden patients and 16 of them also knew that loose clothes should be preferred for such patient (Table 2).

\section{DISCUSSION}

We found that the most common underlying cause of being bedridden was neurological. In a recent study done by Singh et al (2005) also, it was found that the largest contribution to the total morbidity was of neurological disorders. ${ }^{5}$

A majority of the patients took advice from the tertiary care hospitals for their acute care. Most of them also consulted private practitioners for their routine problems. Nine patients were consulting private practitioners exclusively. They preferred private practitioners because they found it difficult to go to the hospital and wait in queue to see the doctor. Moreover, even after waiting for a long time, meeting with the doctor was for a very short time, which left them dissatisfied. Private practitioners were more readily available, gave more personal attention, and were ready to make house calls. Hence, private practitioners share a considerable burden of caring for the disabled. These practitioners can act as a bridge between the tertiary care institutions, which provide acute care, and the long-term care rendered by the families and other informal caregivers.

Most (86\%) of the cases had consulted someone other than the formally trained medical practitioners. They had consulted unqualified practitioners or traditional healers at one point or the other during their course of illness.

In chronic diseases where the focus is not on cure but on limitation of disability and pain relief, the prospect of living in dependency and facing a slow downhill course of health is depressing. So, patients and their caregivers become vulnerable to try out things, which promise improvement without any objective evidence for the same. We came across a wide range of novice treatment modalities for bedsores that were being used in the community. Homemade traditional modalities included til oil (sesame oil), desi ghee (butter), paraffin wax, vinegar and roasted turmeric powder. Neosporin powder and betadine ointment were so commonly used that many caregivers tended to use it for their patients without consulting the physicians. Another modality that held our attention was that of leaving the wound open. The practice of exposing the wound to sun and air existed in many homes. Few other interesting treatment modalities included placentrex gel (fresh human placental extract), bleaching powder and normal saline, 


\begin{tabular}{|c|c|c|c|}
\hline \multirow[t]{2}{*}{ Domains of Knowledge } & \multicolumn{3}{|c|}{ Grading of Knowledge } \\
\hline & Correct & Partially correct & Incorrect \\
\hline - Knowledge of caregivers regarding warning signs of bedsores & 17 & 6 & 19 \\
\hline - To prevent bedsore, a bedridden patient should be & & & \\
\hline dragged slowly for changing the position & 23 & 17 & 2 \\
\hline - Friction can also be a cause of bedsore & 7 & 22 & 13 \\
\hline - Right kind of diet facilitates the ulcer healing & 4 & 5 & 33 \\
\hline - Bedsores are preventable & 9 & 10 & 23 \\
\hline - Overweight patient have lesser chances of developing bedsores & 12 & 5 & 25 \\
\hline - While dressing the wound of the patient hand washing is not & & & \\
\hline necessary if the gloves are worn & 8 & 14 & 20 \\
\hline - Massage is recommended for reddened body areas in bedridden patients & 10 & 6 & 26 \\
\hline - Salt water solution is recommended for cleaning the wound & 10 & - & 32 \\
\hline
\end{tabular}

boric acid, hydrogen peroxide, etc. But the irony lies in the fact that none of these modalities have been tested for its efficacy. People tend to try out whatever new remedy that comes to their knowledge from varied sources. This scenario also indicates that this area has not received desired attention of the medical researcher.

In our study, majority of the cases required assistance while moving or changing position in bed. The frequency of position change was every 2 hourly as recommended in 15 (35.7\%) cases. Three of them were changing the position 4 to 6 hourly and around 4 of them seldom changed it. This can be attributed to the fact that caregiving for family members turns out to be a tedious job. It could be the lack of interest that emerged out of LTC or the belief that the patient cannot recover with mere change in position at frequent intervals. In most of the cases Rexine mattress was preferred over other types. People opting for air mattress usually remained confused regarding the volume of air to be inflated. Singh et al (2005) reported in the study that 19 patients were using water and air beds to avoid bedsores. This was one of the most costly equipments bought for the patient to help in medical care, with alpha beds costing upto $5,800(128 \$) .^{5}$

As expected in the Indian set up, family members were found to be the key caregivers in maximum cases. They had been playing the role of caregivers sans formal training. In a study conducted by Singh et al (2005) also reported that bulk of the cases was provided personal care by their families only. ${ }^{5}$ Help, if any, was hired to perform other household chores. In 54 cases hired help was involved in providing personal care. Of the total 55 hired helps employed, 35 were females. None of these hired helps had received any formal training for case provision. They learnt as they performed under the supervision of the family members. The collective contribution provided by caregivers to support the health and well-being of individuals with disabilities and/or chronic health issues is substantial.
Talley et al (2007) reported that care recipients can be very diverse - in terms of their ages, cognitive disabilities, physical disabilities, mental health needs, and physical/ chronic health needs. ${ }^{6}$

Thus, it is vitally important to be aware of the variety of contexts across the lifespan in which caregiving responsibilities can occur. This is especially true when considering services and the impact of policies designed to capture and assist many types of caregivers as, unfortunately; there are still circumstances that have yet to be recognized appropriately.

Most were caring for their own parents, spouses or siblings, who were bedridden due to conditions such as trauma, cerebrovascular accident, Parkinson's disease and general frailty. They were dependent in various activities of daily living. In a qualitative research by Beth et al (2012), caregiver reports reflected differing levels of engagement and comfort in assisting with caregiving tasks. The variety of tasks was fluid 'depending on the care recipient's diagnosis', and might or might not have included activities and instrumental activities of daily living. The caregivers provided social stimulation, adapting activities to match the care recipients' physical and cognitive abilities. They accompanied the care recipients to medical appointments, communicated with providers, and advocated for the care recipients. They operated and maintained medical equipment such as nebulizers, feeding pumps and wheelchairs. Tasks associated with medical care included changing dressings, wound care, tube feedings and management of 'breathing machines'.

Caregiving tasks were performed in the respective caregiver's, family member' home. Health-promotion activities included medication management, maintenance of a safe environment, skin care, exercise assistance and prevention of bed sores. Caregivers also made decisions on behalf of their care recipients. Frequently, everyday decisions included what to eat or wear, and major decisions 
ranged from what medical care to seek to relocation options once independent living was no longer a safe option.

Average monthly expenditure on the care of the bedridden patient included the payments made to the hired helps and the medicines used for the patient. In 30 of the patients this was between 1,000 and 5,000 which they considered quite affordable on a monthly basis. The expenditure pattern was similar in all the other morbidity groups, i.e. the major part of expenditure was on manpower. Hence in patients needing LTC, it appears that the monthly expenditure depends more on the intensity of needs of the patient and the ability of the family to provide care, and not on the nature of disease. The monthly expenditure rises steeply when the number of hired helps increase.

Another fact that we came across was that when it came to taking financial help from the nonfamily members, hardly $4.8 \%$ of them were availing it from the relatives. According to the caregivers, especially when it comes to financial help, no one really comes forward. Only one person was availing free service from government hospital. This could be attributed to the fact that family members want the best of health care for their loved ones. They hesitate taking up free services. For them money is not an issue, all they want is recovery of the patient at any cost. Majority (69\%) of them had done some sort of modification in their homes. The most common modification was wheelchairs or chairs with commodes, chairs with toilet and bedpans.

Many caregivers were completely unaware of the various warning signs of the bedsores viz pain at the site, areas of duskiness or darkness, discharge, etc. Six of them were partially correct in recognizing the warning signs. This is one reason why caregivers fail to recognize the development of bedsores at the earliest. This leads to the wide prevalence of misbelief among people that bedsores cannot be prevented. At this stage, the need of awareness regarding bedsores is felt because it can affect the attitude and perceptions of the caregivers. Only some of them knew that to prevent bedsore a bedridden patient should be lifted slowly for changing the position. Few of them were in the habit of dragging the patient. This ignorance of caregivers could be due to the fact that LTC of patients renders the caregivers frustrated. Deep within their minds, there is a hidden belief that the patient would not improve. They start thinking that it doesnot make any difference whether they lift or drag the patient.

Very few caregivers believed that friction could be a cause of bedsore development. Shearing forces will only exist if pressure, usually caused by the persons own body weight, is also present. Shear forces occur when a part of the body tries to move but the surface of the skin remains fixed. Friction forces occur when the shearing force increases sufficiently to overcome the body's resistance to movement. Such a movement has an abrasive action. Only four caregivers agreed to the fact that right kind of diet could facilitate bedsore healing. Rest of them could not specify the kind of diet that should be given to bedsore patients.

Doley (2010) reports that increase in protein in the diet by choosing meats, eggs, dairy products, peanut butter, nuts, beans and peas, or protein-supplement powders aids the growth of new cells, collagen and connective tissues. ${ }^{8}$ Bedridden persons are usually malnourished. It may be because of the underlying disease process. Otherwise also, dietary intake of bedridden cases may be less. As a result, there may be less consumption of nutrients especially those which are important for the prevention of bedsore such as protein, amino acids and ascorbic acid, etc.

Few of them believed that overweight people have higher chances of developing bedsores. Majority of them were of the view that overweight had nothing to do with bedsore development. The fact is that obese bedridden people tend to develop bedsores frequently. Reason being adipose (fatty) tissues in small quantities protects the skin by cushioning bony prominences against pressure. However, in moderate to severe obesity, adipose tissues have poor blood supply. This makes the underlying tissues more susceptible to damage. Bedsore may then develop. But contrary to this, there is a study by Compher et al (2007) which states that patients who were underweight had greater odds of developing pressure ulcer; [adjusted odds ratio $(\mathrm{OR})=1.8,95 \%$ confidence interval (CI): 1.2-2.6]. Patients who were obese had reduced odds (adjusted OR $=0.7,95 \%$ CI: 0.4-1.0), and those with severe obesity had the lowest odds of pressure ulcer (adjusted OR $=0.1,95 \%$ CI: 0.01-0.6). This data suggests that extra body fat reduces the risk of pressure ulcers in elderly patients. ${ }^{9}$ Hence this does not apply to bedridden patients of all age groups.

Very few caregivers believed that while dressing the wound of the patient hand washing is necessary if the gloves are worn. That means rest of them was not following the practice of hand washing before and after dressing the wound. Most of them believed that massage is recommended for reddened body areas in bedridden patients. Vigorous skin massage over the bony prominences may lead to deep tissue trauma. This may lead to bedsores. Few of them believed that salt water solution is recommended for cleaning the wound. Many were unaware of the fact that normal saline is used for cleaning most bedsores. It is the preferred cleaning agent because it does not burn or sting the tissue and adequately cleanses most wounds. Not only this, normal saline has multiple uses like it is used as nasal wash in common cold, eye drops contain saline, etc. Moreover it can be prepared at home by dissolving 
approximately half a teaspoonful of table salt into a glass of clean tap water.

On observing the bedsore care practices it was found that correct position was ensured in 37 cases, i.e. large pillows were placed under the head and back and head end of the bed was little elevated in few cases. That means 12\% of the caregivers had no idea as to how a patient on bed for long-time should be dealt with. They left their patient lie in uncomfortable and unrecommended positions that definitely lead to worsening of bedsore status among the patients. Moreover the families who were following the correct positions, they let their patient lie down in the same position for long hours. Hence, following the correct position in such cases made no sense.

Exercises were performed in 16 cases only. Out of those 16, principles of exercises were being followed in 11 cases only. There was a practice of gentle massage among few cases. Regular physical activity causes numerous and substantial physiological performance improving and health-enhancing effects. Vuori (2001) has reported that these effects are seen especially in organs and functions primarily involved in physical activity, i.e. neuromuscular. ${ }^{10}$ But we could feel that caregivers had a laid back attitude when it came to exercises. They preferred hiring a physiotherapist rather than encouraging the patient to perform light exercises.

So, we conclude that the knowledge of caregivers regarding bedsore care practices was inadequate. Many of their beliefs and practices were found to be incorrect. Caregivers try a variety of things for dressing. Lack of proper training in the care of the bedridden leads to low quality of care and high rate of complications in bedridden cases. The caring behaviors among family caregivers of patients with bedsores should be improved. It is necessary to strengthen their perception of the severity and susceptibility of pressure ulcer and thereby to improve their caring behaviors and promote patients' recovery.

There is a need for formal training of the caregivers of bedridden cases with bedsores. We need to evaluate various dressing regimes. A comprehensive policy for care giving to the bedridden should be developed.

\section{REFERENCES}

1. Kutsal GY. Geriatry. Hacettepe Med J 2000;31:40-58.

2. Das SK. Situation analysis of the elderly in India. Report by: Central statistics office ministry of statistics and programme implementation. Government of India 2011 June;30-31.

3. Peterson NC, Bittman S. The epidemiology of pressure sores. Scand J Plast Reconstr Surg 1971;5:62-66.

4. Lyder CH. Pressure ulcer prevention and management. JAMA 2003;289:223-26.

5. Singh T, Singh A, Avasthi A. Pattern of quality of life of long term care for the bedridden patients in Chandigarh, North India. Int J Geriatrics Gerontol 2005;2:1-14.

6. Talley RC, Crews JE. Framing the public health of caregiving. Am J Public Health 2007 Feb;97(2):224-28.

7. Beth M, Smith M, Stanley M. Its like were grasping at anything: Caregivers' education needs and preferred learning methods. Qual Health Res 2012;22:1007-15.

8. Doley J, Mallampalli A, Sandberg M. Nutrition management for the patient requiring prolonged mechanical ventilation. Nutr Clin Pract 2011 Jun;26(3):232-41.

9. Compher C, Kinosian BP, Ratcliffe SJ, Baumgarten M. Obesity reduces the risk of pressure ulcers in elderly hospitalized patients. J Gerontol A Biol Sci Med Sci 2007 Nov;62(11):1310-12.

10. Vuori IM. Health benefits of physical activity with special reference to interaction with diet. Public Health Nutr 2001 Apr; 4(2B):517-28.

\section{ABOUT THE AUTHORS}

\section{Upasana Sharma (Corresponding Author)}

PhD Scholar, Department of Mental Health Education, National Institute of Mental Health and Neurosciences, Bengaluru, Karnataka India, e-mail: usupasanasharma@gmail.com

\section{Sukhpal Kaur}

Lecturer, National Institute of Nursing Education, Postgraduate Institute of Medical Education and Research, Chandigarh, India

\section{Amarjeet Singh}

Professor, Department of Community Medicine, School of Public Health, Postgraduate Institute of Medical Education and Research Chandigarh, India 